\title{
FACULTADES DE LA VICTIMA EN MATERIA PROBATORIA
}

\author{
JaIRo PARRA QUJANO \\ Prolesor de bu Uneridad Eaternide de Colorista \\ Presdema de hribuse lbanamericino de Darecha Precmat.
}

\begin{abstract}
SUMARIO:

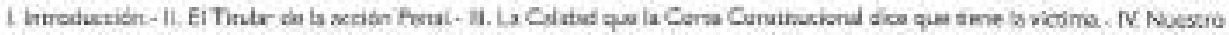

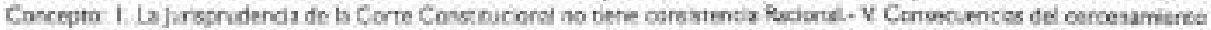

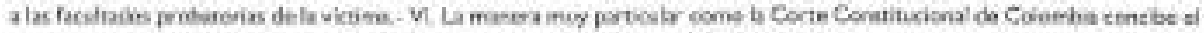
Dericio a li prubs da la vitima. Wi, Condusiones
\end{abstract}

\section{INTRODUCCIÓN}

Para poder ubicar el tema se hace necesario que hagamos uns enunciación (15o un estudio completo), de la participación de la victima en el procese peral, haciendo unas abstracciones de cómo puede ser ella.

En los delitos de scción pública, se puede optar por no darle participación a la victima como interviniente. Pero cuando se concluye que hay que darle participación a la victima como interviniente, se puøde a citula de ejemplo utilizar dos sisternas y el sistema por cierto muy especial que practicamente consig ó ta Corte Constituciond de Colombia.

1) Como cosdyurante del acusador público, es decir, que se puede adherir a la acusación ded fiscal. Puede solicitar pruebas y como es apenas obvio participar en ta practica de las pruebas. interrogar testigos, etc. Siendo adhesivo, no puecie acusar, recurrir in docisión final, ett. Es importante que se retenga que puede pedir pruebas y puede participar en el debate probatorio,

2) Como querellante conjunto. Supone plena sutonomia: puede acusar y er general realizar todos los actos del monopolizador de la acción priblica ${ }^{2}$

3) La victima en Colombia, según la interpretación que de la Constitución hace la Corte Constitudional, no es parte, sino interviniente especia y por ello no tiene las mismas facultades del procesado ni de la Fiscalia. Que su participación como acusador adicional y distinto al fiscal generaria una desgualdad de armas y una transformación esencial de lo que ićentifica a un sisterna adwersarial an la etapa del juicio. En cuano a ba facultades probatorias de la viccima, la Corte Constitucional hace una inkerpretación que de ninguma manera compartimos, sostiene en últimas, que en las etapas anteriores a la dei juicio oral, la victims tiene todas las

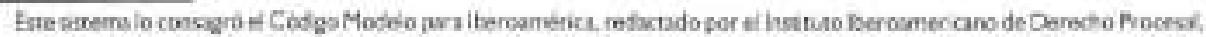
ariedes 269,319 y 291 y rutsingertis

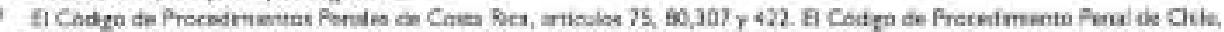

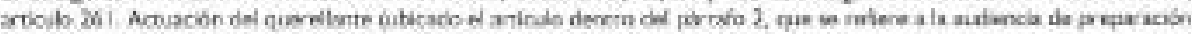

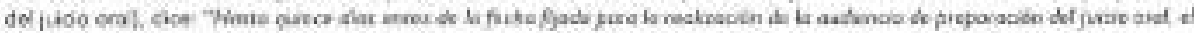
qustelsode por eruiva, podie

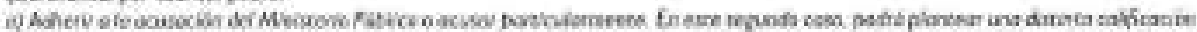

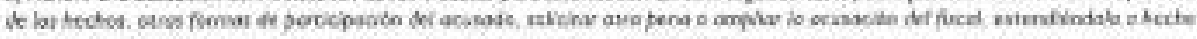

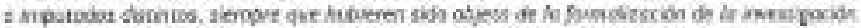

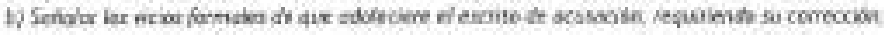

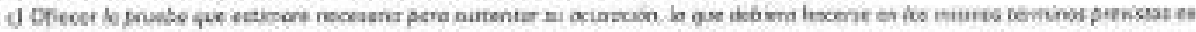
d watiala 259 y

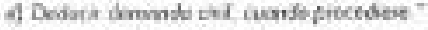

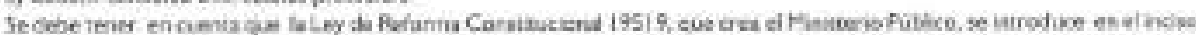

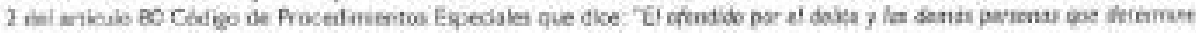

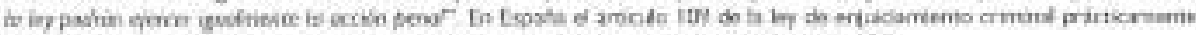

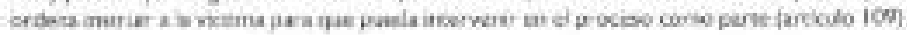


facultades probatcrias, pero en la ecapa del juicio y en cuanto a controverair los mecios de pruaba, los elementos materiales probatorios y evidercia fisica presentados en la ecapa del fisio oral, asi como interrogor al testigo y oponarse a las proguntas que se plantean en el juicio eral, existe ura razón objetiva que impide que la pueda hacer, que no son ocma qua las ya indicidins "'. Puede impugrar la sentencia si está en desacuerdo con ella.

Debe quedar claro, que según la jurisprudencia de la Corte Constitucional Colombiana, la victima no puede participar en el debate probatorio. Por disposición del articulo 443 de la Ley 906 de 2004 , si puede alegar.

\section{EL TITULAR DE LA ACCIÓN PENAL}

Se debe tener en euenta ef articule 250 de la Conatitución Politica de Colombia, fundamentaimente en lo siguiente:

1) La Fiscalia Gerera de la Nación cstí obligada a adolantar el ejercicio de la acción penal y a realizar la invespigación de los hechos.

2) El mumeral 6 del mismo artículo que dice; "Solicitar ante el juez de canocimiento las miedidas judiciales necesarias para lo asistencia a las victimas, to masmo que disponer el restablecimiento del derecho $y$ la reparoción integral a ios ofectados con el uelito",

3) El numeral 7 que dice: "Yelor por la protección de les victimas, tos jurados, las testigos y demús intervinentes en ei proceso perol, ba iey fijard los teminos en que padrón intervenir las victimas en el proceso penal y los mecanismas de justicio restourativa".

De lis anterbor trascripcién, podemos resaltar que se tice: "la ley fijorá las términos en que podrán intervenir las victimas en el proceso penal (...).

La Corstitudión no le dio a la insenvención de la victima ningún calificativo. Solo la llamo interverción, porque el que monopoliza la acción perial es la Fiscalia. Es importante entonces buscar dontro de csa contexto la que significa intervención dentro de un proceso.

Intervención: mostrarse parne en un juicio. Tambidun sobrevenir, en una actividad ya formads. Lungo si la Acción ta monopoliza la Fiscalis. Is intervención, significa parte que sobreviene, en este caso la victima. Una var que ia Fiscalla adejanta el ejarcicio de la acción, puede intervenir ta victima.

Pero siendo la Fiscaliz quien monopoìza fáscción y quien acusa, la intorvención de la victima es voluntaria, es la de une parte condyuvante, es decir, que si intervene es para adherirse a la acuasción de la Fiscriala. Esta concepcibe obsa el reproche que hace la Corte Consxitucionali, esa forma de participación no hace que la victima se convierta en un acusador adicional y distinto del fiscal, que generaria una desigualdad de armas y una transformación esencial de lo suya la pretensión del fiscal."

\section{LA CALIDAD QUE LA COATE CONSTITUCIONAL DICE QUE TIENE LA
VICTIMA \\ 1) En la sentencia C 454 de 2006 y al pronunciarso sobere la contstitucionalidad del articulo 357 de la Ley 906 de 2004, lo declaró exequibla en el entendido que los represencantes de ba victimas}


en el procesa penal, pueden realizar solicitudes probatorias en la audiencia preparatoria, en igualdad de condiciones que la defensa y la Fiscalia.

Y para explicar las razones por las cuales lege a la declaratorla anteries sestiene: "(-.) Ass las casas. enciventra fa Carte que efectivamente ía nurma dernandada incurre en una amisián troscendenze pora el derecho de occeso de la victima a la justicia (Constitución Pafítica artículo 229). en cuanto obstruye sus pasibilidodes de efectiva realizacian de sus derechos a la verdad la justicio y - la reparocián, y le colved, de manera injustificada, en una posición de desventaja en reladión can otros octores e intervinientes"

Los presupuestos que la Corte Constitucional, tiene en cuenta para sostener la inconstirucionalidad por omisión son.

1) El srticulo 357 de la Loy 906 do 2004 , incurre en una cmisión al exciLir del supuesto fáctico, a un sujeto (a vietima), que se encuentra en las mismas condiciones de la Fiscalia, la defersa y aún dal Minststerio Público

1) No existe una razón objetiva y suficiente que excluys a las victimas. Que el modelo procesal diseñado por la Constituciôn considera a la victima coma interviniente. Que se le debe gerantizar su acceso a la justicia (articulo 229 de la Constituciōn Palítica) y de los que de êl surgont ba verdad, la justicia y la reporación.

iii) La omisón genera ura desigualdad injustificada.

iv) La emisión genera el incumplimiento por parte del legislador del deber de configurar una "verdadara intervención", particularmente en la audiencia preparatoria. (Será objeto de estudio mats adelante).

En la sertencia C-209 de 21 de marso de 2007, la Corte Constitucional se pronunció sobre el reconocimienso de la victima come interviniente especial.

Yen esta sentencis en resumen se dijo:

2) Refiriéndoce al sistema que se consagró en la ley 906 de 2004, se dice que se disenó un - esquema prepio para responder a la realdad Colombiana. En otras palabras, derntro del genero sistema acusatorio Colombia elaboró una especie.

Y pera deterninar la forma corno puede actuar la victima en esta especie de sistema acusatorio, sostiene que depende de varios factores:

1) El popel asignado a otros participartes, en particular al fiscal:

2). Del rol que le reconoce la propia censritución at la victima:

3) Del hugar donde ha previsto su participación:

4) De las caracteristicas de cada una de las etapas del proceso penal y.

5) Del impacto que esa participación terya anto para los derechos de la victima come para la estructura y formas propias ded sistema penal acusatorio.

$Y$ en desarrollo de esos factores sostiene:

1) El fiscal es el titular de la acción peral. A ejercer dicha acción, no solo representa los intoresen del Estado, sino también promueve los internses de la victma. La Corte recuerda lo que dice d articulo 250 de la Constitue ón Politica sobre las victimas, y, con relación a la sentencia $C$ B73 de 2003, dce: "Que terrpronamente la Carte subrayó gue ef arriaib 250 de la Cussitución 
no supedita a los victimas a recibir la proteccolón dei fiscai, exchuswampente, sine que reconsce que eflas pueden intervenir en el proceso penal y canfía al legisladar descrrallar dicha posibilidad".

2) Con rolación al rol que le reconoce la Constituxión a la victima, la Corte cita la sentencia C. 59! de 2005. y en ella se dice: "la victima, a so vez tiene derecho a canocer to verdad. occeder a lo administración de justicho, a la reporación integral, csi como a cobtener medidos judicioies de procecsión, sin perjuicio de peder acudir ante la jurisưucción cinil ordinaria paro efectas de abtener le reperación del dario, ocusionodo con el delito. La intervención de la victima en el proceso penal, constitioge otra de las particularidades de nuestro sistema precesal penal".

3) La Corte cita el articulo 250 de la Constitución $x$ concretamense los numerales 6 y 7 para referirse al papel cue cumple el fiscal frente a las victimas dentro de esta especie de proceso penal.

4) Según la Corte Consticucional, el numeral 7 del articuío 250, esboma los rasgos bisicos del rol que cumplen las victimas dentro del proceso: i) Tienen el caracter de intervinientes. 11) La intervención de las víctimas se ejerce de manera sutónoma de las funciones del fiscal; aj) No supedita la noma constitucional la intervención de las viktimas a la actuación del fiscal; iv) EI logislador debe decir cómo intervendrán las victimas en el procaso penal. v) La intervención de las victimas se da en todo el proceso panal. Pero la Corte agrega: "Sin embargo, tal posibilided a de ser armónica con le estructura del proceso acusatorio, su iógica propia y la proyección de le misma en cada etapa del proceso:.

Además agrega la Carte: "De lo anterior se concluye que la victima del deñito no es un sujeto pasivo de protección por parte do is Fistalia, sino un interviniente octivo, constitucionaimente iegitimado para hacer valer sus dorechos denuro del proceso penal instaurado per el acto 03 de 2002 y la Ley 906 de $2004^{*}$.

Tambiòn dice k Corte Constitucional:

'Se resalto, no obstonte. que los derechos especificas que se ie reconocen e la victima no le quitas su caràcter de intervinieste, sho que la proyecten comso una fieura especial en las distianas ecopos din froceso penuf de tenuencia acusataria, para que haga voler sus derechns a la verdad, Jo justicio y la reparación integral. So interrención no se circturscribe o uno perticipoción find en ef incidente de reparocich uno ver cancluido ef juiclo, ya que ello no se campodece con lo seriniado en el articulo 250 citado, $y$ significario une restncciön de sus derechos a ia verdad $y$ a fo jasticia puesto que la victima participaría octivamente no salo a efoctos de exigir reparación".

5) Esse sparte se podria denominar esquema procesal y la participación de la victima Se hace necesario hacer una dita extersa de lo que dice la Corte Constitucionat:

"En este mevo exquerno penal de cendencio acusotoric el cosstituyente montuvo io disbinción entre ia fase de investigación -encaminada a determinar si hay méritos pare ocusar- y la fase de jiagamienco $y$ otorgó una clara prepondérancia a esta úitime, corstitugendola en el cantro de gravedod del proceso penal bojo ef sistema instituido por

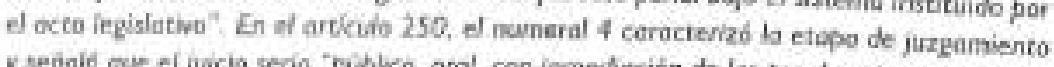

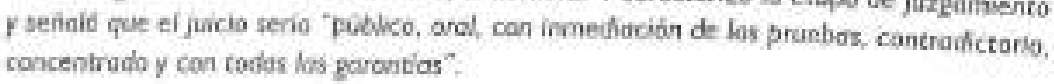

"La definición y curactenzación de las distintros etapos del procese pend (investigoción.

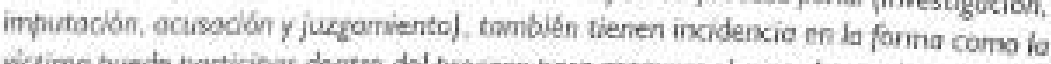
nictime puede porticipar dentro del proceso para osegurur el goce de sus darechos a la 
verdad, la justicia y la reparación. Resaito fa Corte que solo respecto de la etapa del juicio, el constitujerite precisó sus cardacter/sticas, eñfatizundo su carócter adversorial. asi no hayi seguido un modelo puro en este aspecta. Este hecho tiene incidencia en la forma carnu pueden actuar las victimas durante esta etapa. Como quiera que este carácter adversarial supone la confrontoción entre el ocusado y ei acusador, la posibilidad de octuación directa y separada de las victimas, al margen del fiscal, se encuentra restringida por el propio texto constitucional que definió los rasgos del juicio".

\section{Dics igualrsente la Corte:}

Se pregunta entonces fa Carte Constitucianal, si dado que nuestro sistema penal tiene elementos distintivos ton particulares y propios. la participoción de la víctine en coda una de las etapas procesains debe tener las coracteristicas de un interviniente especial o de una parte procesal camo alega el accianiente.

En primer hagar, considera esta Corporoción que si bien la Constitudión previó la participación de fo wictimo en el proceso penal, no le otorgó lo condición de parte, sino de interviniente especiol. La asignación de este rol particular determina entonces, que la victima no tiene las mismas facultodes de/ pracesodo ni de la Fiscalia, pero si tiene algunas capocidodes especiales que le permiten intervenir activamente en el proceso penol".

6) Este aparte se podria denominar las etapas procesales y el impacto que tondria la participación de la victima.

\section{La Corte Conatitucional dice:}

"En segundo irgar, dado que el canstitiyente definio que la wictima podrí interveniv a to larzo del proceso peral, es preciso tenev en cuenta los elementeas especificas de codo etapa procesaf y ei impocto que tendría lo porticipeción de fo victima en coda una de ellos. En ese conterto, es necesporio resaltar que cuundo el canstituyente definio que lo etopa dei juicio tuviera un carócter adversarial, enfetizí ius especoficidades de esa confrontoción entre das partes: El acusador y el acusndo, úfiando de farlo ia pasibilidad de canfrontación de varios acusadares en conva del ocusado. La aralidad, in inmedioción de priebos, fa controdicción $y$ las garontlas del procesado se fegran de manero adecuada si se preserva ese cardicter adversarial. Aar el contravia fa particpacián de la victima camo acusodar adicionol y distinto al fiscai generaria une desigualdod de armas $y$ uno trinafarmoción esencial de lo que identifica un sistemu adversarial en lo etapa del juicia. Por otro porte, el constituyente no fjó las caracteristicas de las demás etapos del proceso penal, y per lo tanto, delegó en ef iegisfodar la focultod de configurar esas etopes procesales.

De la enterior, surge entences que fos efementos definvterios de ia participacian de fia victima como interviniente especial en las dfferentes etapos del proceso penol depente de la etapa de que se vate. $x$. en esa medido, la pasibilidad do intervencián es mayor en las etapas previas o posteriores el juicio y mienor en fa etapo dai juicio-

En desarrolio del ancerior plantearrienta, la Corte Constitucional, se pronunció scobre algunas etapas del proceso:

Las pruebas anticipadas, consagradas en el numeral 2 del articulo 284 de la Ley 906 de 2002. En esa norma se debe tener por induida a la victima, como sujeto logitumado para selicitar th prumba anticipadas. Excluir o pretermitir a la victima, la impide asegurar el derecho a la 
verdad y dé derecho de las victimas consagracio en el litaral d) del artículo II de la Ley 906 de 2004, es decir. "A ser aidas y a que se fes facilite ell abarte de pruebas".

i) En el articulo 344 del Código de Procodimiento Panal, debe entenderse incluida la victima como sujeto legitimado para solicitar al descubrimiento de un elemerto material probatorio especifico o de evidencia fisica especifica.

iii) En el articulo 356 del Codigo de Procedimiento Panal, Sebe entendersa includa ta victima como sujeto legitimado, para hacer observaciones sobre al descubrimiento de elementos probatorios y do la tocalidad de las pruebas que se harán valer en lo audiencia del fuicio oral.

iv) En El articule 358 del Codigo de Procedimiente Penal, se dobe tener como sujeto legitimsdo, para solikirar la exhibición de los elementes materiales do pruaba a la victima.

v) En el inclso primero dal articúa 359 del Cádigo de Frocadimiento Ponal, debe entenderse incluido como sujeto legitimado a la victima, para solicitar la exclusión, el rechazo o ia inadmisiblidad de los medios de pruebe.

vi) En relación con el articulo 378 y el articulo 391 del Código de Procedimiento Penal, que excluyen a la victima de los actores procesales que pueden controvertir los medios de prueba. los elemantos mareriales prabatories y la evidencia fisica presantadas en la etapa dal juicio oral, asi como interrogar al testigo y oponerse a las preguntas que se planteen en el juicio oral;

wi) Sin errbargo, dada que la posbilidad de ejercer estas facultades ociare en la ctapa del juicio oral, ti oxitte una razón objetina que justifica la limitación de los derechos de la victima como quiera que su participasión directz on al juicio oral implè ura modificación de los rasgos estructurales del essema peral acisatorie que comporta una alteración sustancial de la igualdad de armas y convierve a la victima en un segundo acusador o contradictor an desmedro de b dimenskn adversarial de dicho proceso.

Far ello, este amisión no genera uno desigualdad injustificodo entere lon distintos octores dei proceso penol, sino que busca evitac que la defensa quade en una situoción de desventaja en el juicio and dados sias rospos esersciafes definidas por ef propio constituyente; $y$.

Tampoco supove un incumpliminte por parte del legisiador del deber de configurar wia intervenoion electiva de la victima en el frocesa penal, camo quiera que la pasibuidod de que lo victima (o su opoderada). intervenga para controvertir los medios de pruebo, los dementos inoteriales probatarios y la evidencio física presentadus en la etapo del jaicio arol, asi come interrogor al testigo y oponerse o fas preguntes que se planteen en ei juicio wal, se ejercerá a través del fiscal con base en la acthidad propia y en la de las víctimas en las etapas previas del proceso, segün los derechos que le han sido reconocidos en esta sentencia y en la ley. En efecto, a lo largo del proceso penal, en las etapas prevlas, la victima ha podido participor como interviniente especial en la construcción del coso para defender sus derechos, de tal forma, que en el juicio mismo estos ye proyectarán medlante la actividad del fiscof".

No obstante, la victime a travós de su abogado, podrá ejercer sia derechos en la etapa del jucio sin convertirse en una parte que pueda presentar $y$ defonder su propio caso al margen del fiscal. El conducto para culminar en esca etapa final del proceso el ejercicio de sus darechos es el fiscal, quien debe oir al abogado de la victima. Ast. por ejemolo, este podra. aportar a la flacilíla observociones para facilitar la contradicción de las alementos probatorios, artes. 
y durante el juicio oral, pero solo el fiscal tendrá voz en la audiencia en aquellos aspectos regulados por las normas acusadas. En el evento de que la vietima y su abogado están en desacuerdo con la serntencia poidrin ejercer el derechs da impugnarla, de conformidad con al articulo 177 de la Ley 906 de 2004 .

\section{NUESTRO CONCEPTO}

Para que haya un poco de clandad precendo sintetizar mis planteamientos en los siguientes puntos:

1) Es cierto, como lo dice la Corte Constitucional y teniendo en cuenta el numeral 4 del articulo 250 de la Constitución Política que alfi se consagró un sistema acusatorio, peré en nirguna parte se consagró un proceso bipolar, competición de dos partes y aín aceprando en vía de hipótesis que ello fuera cierto, no es acertado lo que dice la Corte Constitucional "de modo que fo introduccidin de un tercer actor en an htigio de este tipo puede afectar odversamente los incentives requeridos para sustestanlo". No hay un tercer actor, porque la victima no involucra una presensión, sino que hace suya la pretensión de la Fiscalia, con el fit de acceder a ta verdad, justicia y reparación. Cosa distinta es que se pretendiera que la víctima pudiera hacer stu propia acusación o anexar una acusación a la formulada por la Fiscalia. Solo se pretende que interverga en la prictica de las pruebas.

ii) También se debe tener en cuenta que la victima no es sujeto legitimado para incoar la acción penal. y por tanto, no puede por ejemplo; acusar, etc.

ii) El numeral 7 del articulo 250 de la Constituoión Política, no limita la intervención de las vicuimas y menos cuando se tiene claro que ellas ne pueden imolucrar presensiones (acusación) distintas a las de la Fiscalia So preseritar a intervenir en la práctica de las pruebes. La limitación establecida genera una desigualdad injustificada e irrazorable. Es un verdadero desaguisado que se le permita a la victima particlpar en la producción del caso (en las etapas previas al juício), pero no en la práctica da las pruabas, que es la verdadera esencia de la pruaba. La prueba es dialéctica.

iv) Siguiendo con el silenciamiento de la victima para ejercer el derecho de contradicción, se pretende que ésta lo ojarza, haciéndole observaciones al fiscal. El fiscal será el diafragma de la victima. Esta facultad, si así se puede llamor le resta dignidad a la victima. (Más adelante se desarrollari este plarneamiento).

v) Al haber el constitupente establecida en el numeral 7 del articulo 250 de la Constitución Política, que "ia Ley fjard ios témines en que podrón intervenir las vietimas en el proceso penaf y los mecanismos de justicio restaurotino". Consagró la intarvención de las victimas. ¿Qué clase de intervención? Respondarnos: Una intervención Adhesiva. ¿Por qué aulhesival Rexpondamos Porque al monopolzar el fiscal la acción porral y además al habor radicado ta precensión (acusación) el constituyente en cabeza del fiscal (numeral 4 del articulo 259), a victima, desde el purnto de vista constitucional, no puede protender (acusar ni separada, ni conjuntamerte con fiscal). luego siendo interveniente. puede azudar a hi parte que acusat, mediante una detcrminada actividad para el mayor exita de ella. Fuede realizar probatoriamence lo permitido a la defensa y a la fiscalia, sin que se pueda negar la intervención en la audiencia oral, donde no enriquece la relación ya que no acusa, como quedo dicho.

Ahora, en forma separada haremes unas consideraciones sobre algunos aspectos relerantes.

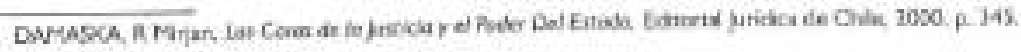




\section{La luriserudencia de la Corte Constitucional no tiene consistencla racional}

No es consistente que se le percrita a la victiru participar en la audiencia preparatoriay hacer solicitudes prabatorias (so debe entender que inwestigaron fuentes de pruebs, entrevistaron teatigos). y que luego cuando se trata de ta recepolon de medies de orueba que tienen fuentes de prueba investigadas y ubicadas por la victima, esta no pueda intervenir en su recapoión y contradiceión. Ese cercenamiento a sa intervencikin lo sonvierte prácticamente en rey de burlas, porque sin dialéctica no hay pruebas.

La victima, cuando puede intervenir en el juicio oral, se dignifica 'y io participocián de quienes han sufinido of doflo provocodo por un delincuente, puede ofrecer lo dimensión humano que sensibilizo of púbíce hacie las abjetivos del Estada. incrementando su poder oficial para implementar polízicas mediante et espectócialo del juicio".

El manejo de la audiencla con la presencia de la victima no vene ningún problema, por ejermplo: si se trata de ia recepción de la declaración de un testigo poatulado por la victima, esta (cl apoderado) interrogará, mediante el llamado incarrogatorio directo, $y$ después, las demás partes podrán formular los contrainterrogatorios y asi sucesivamente con los dernis medios de prueba. dalensa.

Igusimente, con respecto a los demis medios de prueba sobre todo los postubdos por la

\section{CONSECUENCIAS DEL CERCENAMIENTO A LAS FACULTADES PROBATORIAS DE LA VICTIMA}

1) Arriesga el prestigo del fiscal o por lo menos puede dar lugar a nanores sobre su idoneidad. Sa el acusado resuta ahsuelto y la victima cree que con las fuettes de prucba que teria hubiera logrado la condena si so le hubiera permitido participar en el debate. El fracaso por decirlo en alguna forma se lo kvemarian al fiscal.

ii) SEla victima ha entrevistado un testigo, por ejemplo, para postularlo como medio de prueba. eso que percibió la victima que pueda ser útil para el interrogatorio no es transmisible al fiscal por lo que ta Corte Constitucional lama: "observacianes pora focilitar ía contraticción de los elementas probotorins"

La jurisprudencia de la Corte Constitucional soloca a la victima en unz actividad rogativa yle quita dignidad.

No cabeduda que la victira, como ya se sosturo no es parte principal, es como ya ve demostro, una parta coadyuvante y sid de conformidad con la jurisprudencia de la Corte Constitucional piseden frecer solicitudes probatorias en la audiencia preparatoria, dándosele un trato justo, cuando se lo excluye de la facultad de participar en la contradicción de la prueba y se le sustituye por hacer abservaciones a la Fiscala, se le quina la dignidad y no cabe duda que se interpreta mal la Constitución por parte de ls Corte Comstitucional.

\section{LA MANERA MUY PARTICULAR COMO LA CORTE CONSTITUCIONAL DE COLOMBIA CONCIBE EL DERECHO A LA PRUEBA DE LA VICTIMA}

Ha dicho la Corte Conseitucional:

La efectividad del derecho a decoeder a la justicia, en el pae se inscriben las derechios a ie verdat, a la justcio y a in reperociän de las victimas, so encuentra en una nefoción directo con

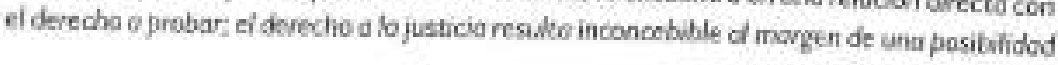


real de incidir probatariamente en el esclarecimiento de las hechos $y$ en ia determinacián de responsibbildades; $y$ ef derecho a la reporacián, cuando se ejerce en el proceso penal, se consalida a partir de lo determinoción de la respansobilidad par el hecho purible"?

Las facubades que se le han otorgado a la victima, teniendo en cuenta lo que ha sostenido is Corte Carsteucional, resultan siendo un simple sedante, si no se le penesite participar en la prácticay conaradicoísn de la prueba an la audiencia oral. Con esa prohíbición se niega al derecho a la prueba y se contradice lo que dice la misma Corte Constitucional, en lo que aparece traserizo, ya que se lo priva del derecho a "incidir en forma plens en el esclarecimierto de los hochos".

\section{CONCLUSIÓN}

El proceso penal colombiano no es bipolar como ya se dijo anteriormente, sino una especie del acusatorio, hasta el punto que el Ministerio Publico interviene en el juicio y, en una forma vital, con iniciatha protatoria, como aparece reglado en el inciso 4 del articulo 357 de la Ley 906 de 2004, donde se lee: "Encepeianalmente, ogotodes las solicitudes probotovias de las portes, si el Miristerio Aublico tuviere conocimiento do le exsistencie de und pruebo no pedida par estas pue pudiera tener esencial infiuencio en los resuftodos del juicia, solicitord su próctica". Adernis el Ministeria Püblico podrá interragar a los testigas, a los peritos, para el cabd encendimiento del asunto. El Ministerio Púbàco, podra cponerse a la preguna del interrogador (articulo 395), etc. No rompe el equilbrio procesal, permitir que la victima participe en el debate probatorio en el fuicio oral. Sin este derecho no hay derocho a la prueba. 